This item was submitted to Loughborough's Research Repository by the author.

Items in Figshare are protected by copyright, with all rights reserved, unless otherwise indicated.

\title{
Solitary waves of a coupled Korteweg-de Vries system
}

PLEASE CITE THE PUBLISHED VERSION

\section{LICENCE}

CC BY-NC-ND 4.0

\section{REPOSITORY RECORD}

Grimshaw, Roger H.J., and Gerard looss. 2019. "Solitary Waves of a Coupled Korteweg-de Vries System". figshare. https://hdl.handle.net/2134/706. 


\title{
Solitary waves of a coupled Korteweg-de Vries system
}

\author{
Roger Grimshaw ${ }^{a *}$ and Gerard $\operatorname{Iooss}^{b}$ \\ ${ }^{a}$ Department of Mathematical Sciences, Loughborough University, Loughborough, \\ LE11 3TU, UK \\ email R.H.J.Grimshaw@lboro.ac.uk \\ ${ }^{b}$ Institut Universitaire de France, INLN, UMR 129 CNRS-UNSA, Sophia-Antipolis, \\ 1361 route des Lucioles, F 06500 Valbonne, France \\ email: gerard.iooss@inln.cnrs.fr
}

\begin{abstract}
In the long-wave, weakly nonlinear limit a generic model for the interaction of two waves with nearly coincident linear phase speeds is a pair of coupled Korteweg-de Vries equations. Here we consider the simplest case when the coupling occurs only through linear non-dispersive terms, and for this case delineate the various families of solitary waves that can be expected. Generically, we demonstrate that there will be three families, (a) pure solitary waves which decay to zero at infinity exponentially fast, (b) generalized solitary waves which may tend to small-amplitude oscillations at infinity, and (c) envelope solitary waves which at infinity consist of decaying oscillations. We use a combination of asymptotic methods and the rigorous results obtained from a normal form approach to determine these solitary wave families.
\end{abstract}

Keywords: solitary waves; Korteweg-de Vries equations; normal forms.

*Corresponding author

\section{Introduction}

It is well-known that the Korteweg-de Vries equation is the generic outcome of a weakly-nonlinear long-wave asymptotic analysis of many physical systems. It is categorized by its family of solitary wave solutions, with the familiar "sech" ${ }^{2}$ " profile. However, in those circumstances when there are two wave modes with nearly coincident 
linear long-wave speeds, the generic outcome is a set of coupled Korteweg-de-Vries $(\mathrm{cKdV})$ equations. In the simplest case, these take the form,

$$
\begin{aligned}
& A_{t}+\Delta_{1} A_{x}+\mu_{1} A A_{x}+\lambda_{1} A_{x x x}+\kappa_{1} B_{x}=0 \\
& B_{t}+\Delta_{2} B_{x}+\mu_{2} B B_{x}+\lambda_{2} B_{x x x}+\kappa_{2} A_{x}=0
\end{aligned}
$$

Here $\triangle_{1}-\triangle_{2}$ is the detuning parameter which measures the difference in the linear long-wave speeds of the uncoupled system, $\kappa_{1}, \kappa_{2}$ are the coupling parameters, while $\mu_{1}, \mu_{2}$ and $\lambda_{1}, \lambda_{2}$ are nonlinear and linear dispersive coefficients respectively. Note that the coupling here occurs only through linear non-dispersive terms; more generally the coupling may occur through linear dispersive terms and through quadratic nonlinear terms as well. Such systems have been derived in several geophysical flows (see, for instance, the recent review [11]).

This brief article has two objectives. The first is to describe the three families of solitary waves that can be expected, namely (a) pure solitary waves which decay to zero at infinity exponentially fast, (b) generalized solitary waves which may tend to small-amplitude oscillations at infinity, and (c) envelope solitary waves which at infinity consist of decaying oscillations. The second objective is to indicate how the normal form approach, which exploits dynamical systems theory, can be used to make this description rigorous.

\section{Linear dispersion relation and solitary waves}

The linear dispersion relation is obtained by omitting the nonlinear terms in $(1,2)$, and then seeking solutions proportional to $\exp (i k(x-c t))$. The result is,

$$
\left(c-\Delta_{1}+\lambda_{1} k^{2}\right)\left(c-\Delta_{2}+\lambda_{2} k^{2}\right)=\kappa_{1} \kappa_{2} .
$$

Also note that the linearized system is spectrally stable if the relation (3) yields only

real values of $c$ for all real wavenumbers $k$. It is readily verified that this is so if either $\kappa_{1} \kappa_{2}>0$, or if when $\kappa_{1} \kappa_{2}<0$ then $\left(\Delta_{1}-\Delta_{2}\right)^{2}+4 \kappa_{1} \kappa_{2}>0$ and $\left(\Delta_{1}-\Delta_{2}\right)\left(\lambda_{1}-\lambda_{2}\right)<0$; otherwise there is a band of instability in $k$-space for a finite range of wavenumbers $k$, which may include $k=0$.

This dispersion relation can take several forms, depending on the relative signs of the coefficients $\Delta_{1,2}, \lambda_{1,2}$ and $\kappa_{1,2}$. In figure 1 we show three typical scenarios. In each case we take $\Delta_{1}=-\Delta_{2}$ where $\Delta_{1}>0$ without loss of generality, and we take $\kappa_{1,2}>0$. Then, in figure 1a,b,c we show the cases when (a) $\lambda_{1}, \lambda_{2}>0$, (b) $\lambda_{1}>0>\lambda_{2}$, and (c) $\lambda_{1}<0<\lambda_{2}$ respectively. In each case the dispersion relation contains two branches, which we label as the $A, B$-branches respectively. The $A$-branch ( $B$-branch) is that which can be identified with the $A$-wave ( $B$-wave) in the limit when the coupling 
parameters $\kappa_{1,2} \rightarrow 0$. With $\Delta_{1}>0$ the $A$-branch $(B$-branch) is the upper (lower) branch in figure 1 . Note that, in relation to the normal form analysis of Section 3 , figure $1(\mathrm{a})$ corresponds to figure $2(\mathrm{a})$ and figures $1(\mathrm{~b}, \mathrm{c})$ to figure $2(\mathrm{c})$.

From these dispersion relations we can anticipate the existence of three possible kinds of solitary waves for which $A=A(x-c t), B=B(x-c t)$, namely, pure solitary waves (PSW), generalized solitary waves (GSW) and envelope solitary waves (ESW). In each case, the bifurcation point is identified as that value of wavenumber $k$ for which the $c=c_{g}$ where $c_{g}$ is the group velocity, given by $c_{g}=c+k(d c / d k)$; thus, the bifurcation point is where $k d c / d k=0$. Hence, there is always a bifurcation at $k=0$ leading to either pure or generalized solitary waves. There may also be bifurcations at finite, non-zero wavenumbers $k$ leading to envelope solitary waves. A rigorous approach using normal form analysis to describe these bifurcations is presented in Section 3. Here, we present an alternative (and non-rigorous) approach which uses weakly nonlinear asymptotic methods

Pure solitary waves: These bifurcate from a dispersion curve branch at wavenumber $k=0$ in the sense opposed to that of the linear dispersion curve, for the case when there is no possible resonance with the other branch for any wavenumber $k$. Thus they can be found in figure 1 a by a bifurcation from the $A$-branch, and in figure $1 \mathrm{c}$ by a bifurcation from both the $A$-branch, and the $B$-branch. In the weakly-nonlinear long-wave limit these solitary waves can be constructed by an asymptotic expansion, which in effect leads to a Korteweg-de Vries equation. First note that in the linear long wave limit the phase speeds determined from (3) are given by $c= \pm c_{0}$ where $c_{0}=\left\{\Delta^{2}+\kappa_{1} \kappa_{2}\right\}^{1 / 2}$. Suppose the bifurcation takes place from the $B$-branch of figure $1 c$, and so takes place from the point $c=-c_{0}$. Then let

$$
(A, B)=\epsilon^{2}\left(A_{1}(X), B_{1}(X)\right)+\cdots, \quad c=-c_{0}+\epsilon^{2} c_{1}+\cdots, \quad \text { where } \quad X=\epsilon(x-c t) .
$$

It is readily found that, at the leading order, $\mathrm{O}\left(\epsilon^{2}\right),\left(A_{1}, B_{1}\right)=\left(-\kappa_{1}, c_{0}+\Delta\right) S(X)$. At the next order, $\mathrm{O}\left(\epsilon^{4}\right)$, a compatibility condition is needed on the equations for $u_{2}, v_{2}$ which leads to the determination of $S(X)$,

$$
\begin{array}{r}
S=a \operatorname{sech}^{2} \gamma X \\
\text { where } \mu a / 3=4 \lambda \gamma^{2}=c_{1}\left\{\left(c_{0}+\Delta\right)^{2}+\kappa_{1} \kappa_{2}\right\}
\end{array}
$$

where $\mu=\left[\mu_{2}\left(c_{0}+\Delta\right)^{3}-\mu_{1} \kappa_{1}^{2} \kappa_{2}\right]$, and $\lambda=\left[\lambda_{2}\left(c_{0}+\Delta\right)^{2}+\lambda_{1} \kappa_{1} \kappa_{2}\right]$. This leading-order result is easily recognised as just the well-known Korteweg-de Vries solitary wave. The expansion can, in principle, be carried through to higher orders. This procedure is very well-known and has been used in many physical problems (see for instance, [1] for water waves, or [12] for internal waves ).

Generalized solitary waves: These bifurcate from a dispersion curve branch at wavenumber $k=0$ in the sense opposed to that of the linear dispersion curve, for 
the case when there is possible resonance with the other branch for some finite nonzero wavenumber $k$. Thus generalized solitary waves can be found in figure 1a by a bifurcation from the $B$-branch, and in figure 1 b by a bifurcation from both the $A$-branch, and the $B$-branch. As for pure solitary waves in the weakly-nonlinear long-wave limit these solitary waves can be constructed by an asymptotic expansion, as above leading to a "sech" structure at the leading order, but this now needs to be supplemented by a calculation of the oscillations which persist at infinity. This usually requires the use of exponential asymptotics (see, for instance, [4], [6] or [13]).

Here, we note that a simple alternative to directly invoking exponential asymptotics is to assume that $\kappa_{1,2}$ are small parameters, and then perform an expansion in these parameters (for a detailed discussion of this approach, and a comparison with the method of exponential asymptotics in a related problem, see [13]. Let us suppose that the bifurcation takes place from the $B$-branch of figure 1(a). Again we assume that $A=A(x-c t), B=B(x-c t)$ and let $\xi=x-c t$. Then we see that $A$ is $O\left(\kappa_{1}\right)$ and that, to leading order, $B$ satisfies the steady version the Korteweg-de Vries equation, and so, $B=B_{s}+O\left(\kappa_{1} \kappa_{2}\right)$ where $B_{s}=a \operatorname{sech}^{2} \gamma \xi$ and $c+\Delta=\left(\mu_{2} a\right) / 3=4 \lambda_{2} \gamma^{2}$. Next, $A$ is given by

$$
A_{\xi \xi}+m^{2} A=-\frac{\kappa_{1}}{\lambda_{2}} B_{s}
$$

where $\lambda_{1} m^{2}=\Delta-c>0$, readily satisfied here since we expect $c$ to be close to $-c_{0}$. Equation (7) is readily solved explicitly. Seeking solutions which are symmetric in $\xi$, we find that, as $x \rightarrow \infty$ oscillations persist at infinity, whose amplitudes may be explicitly calculated. For small amplitudes of the solitary wave core $\gamma \rightarrow 0$ and these oscillations have amplitudes which are then exponentially small with respect to $\gamma$.

Envelope solitary waves: These arise here as a bifurcation from points on a dispersion curve where there is a minimum or maximum value of $c$ for a finite non-zero value of $k$. As noted just above, the significance of such points is that they are locations where the phase speed $c$ and the group velocity $c_{g}$ are equal, thus leading to structure where the phase and the envelope of a wave packet can propagate at the same speed (such waves were rigorously obtained found for capillary- gravity waves in [7]). They can be identified in figure $1 \mathrm{~b}$ as a bifurcation from both the $A$-branch, and the $B$-branch. Again, a weakly nonlinear asymptotic expansion can be used to construct these envelope solitary waves (see, for instance, [2]), The asymptotic approach assumes that at leading order the solution for $A, B$ has the form $(A, B) \sim \epsilon\left(A_{1}(X, \tau), B_{1}(X, \tau)\right) \exp \left(i k(x-c t)+\right.$ c.c. where $X=\epsilon\left(x-c_{g} t\right)$ and $\tau=\epsilon^{2} t$, and c.c. denotes the complex conjugate. Here $k$ is that wavenumber where $d c / d k=0$, and so $c_{g}=c$ there. The expansion procedure leads to a nonlinear Schrodinger equation for $A_{1}$ (or $B_{1}$ ), and with the inclusion of some higher-order terms, steady solutions of this equation yield the desired construction of an envelope solitary wave. We shall not give details here, but see [5] where this approach was carried out and compared with the normal form analysis. 


\section{Normal form approach}

First we look for travelling wave solutions of $(1,2)$, that is, we assume that $A, B$ are functions only of $\xi=x-c t$, then integrate once, and set the integration constants equal to 0 . The result may then be written as a dynamical system for $(A, B, C, D)(\xi)$,

$$
\begin{aligned}
& A^{\prime}=C, \\
& C^{\prime}=\lambda_{1}^{-1}\left[\left(c-\Delta_{1}\right) A-\frac{\mu_{1}}{2} A^{2}-\kappa_{1} B\right], \\
& B^{\prime}=D \\
& D^{\prime}=\lambda_{2}^{-1}\left[\left(c-\Delta_{2}\right) B-\frac{\mu_{2}}{2} B^{2}-\kappa_{2} A\right] .
\end{aligned}
$$

Here $A^{\prime}$ etc. denotes the derivative with respect to $\xi$. Importantly, the system is reversible in that the vector field anticommutes with the symmetry $(A, B, C, D) \rightarrow$ $(A,-C, B,-D)$. Note that if instead we were to choose to take arbitrary values for the constants of integration, then there would be two additional parameters. This case is left for further study.

Let us denote the unknown vector function by

$$
U=(A, C, B, D)^{t}
$$

then, the linearized operator obtained from (11) reads

$$
L=\left(\begin{array}{llll}
0 & 1 & 0 & 0 \\
\lambda_{1}^{-1}\left(c-\Delta_{1}\right) & 0 & -\lambda_{1}^{-1} \kappa_{1} & 0 \\
0 & 0 & 0 & 1 \\
-\lambda_{2}^{-1} \kappa_{2} & 0 & \lambda_{2}^{-1}\left(c-\Delta_{2}\right) & 0
\end{array}\right) .
$$

Defining $\delta_{1,2}=1 / 2\left(\Delta_{1} \pm \Delta_{2}\right)$, and considering the parameter plane $\left(\delta_{2}, c\right)$, fixing $\delta_{1}$ and the other parameters, the dispersion relation (3) takes the following form,

$$
\lambda_{1} \lambda_{2} k^{4}+(D) k^{2}+(H)=0,
$$

where

$$
\begin{aligned}
(H) & \equiv\left(c-\delta_{1}\right)^{2}-\delta_{2}^{2}-\kappa_{1} \kappa_{2}, \\
(D) & \equiv\left(\lambda_{1}+\lambda_{2}\right)\left(c-\delta_{1}\right)+\left(\lambda_{1}-\lambda_{2}\right) \delta_{2} .
\end{aligned}
$$

Note that here the dispersion relation has been written as an equation for $k$ with $c$ given, where the eigenvalues $\sigma$ of the linearized operator $L(13)$ are such that $\sigma=i k$.

The discussion for the location of the eigenvalues $\sigma=i k$ in the parameter plane $\left(\delta_{2}, c\right)$ is driven by three important simple curves, given by,

$$
(H)=0, \quad(D)=0, \quad(P)=0,
$$


where

$$
\begin{aligned}
(P) & =(D)^{2}-4 \lambda_{1} \lambda_{2}(H) \\
& =\left[\left(c-\delta_{1}\right)\left(\lambda_{1}-\lambda_{2}\right)+\left(\lambda_{1}+\lambda_{2}\right) \delta_{2}\right]^{2}+4 \lambda_{1} \lambda_{2} \kappa_{1} \kappa_{2} .
\end{aligned}
$$

(i) The curve $(H)=0$ defines the long-wave limit $k=0$, which here, in the $\left(\delta_{2}, c\right)$ plane is a hyperbola centered at the point $\delta_{2}=0, c=\delta_{1}$.

(ii) $(D)=0$ is a line passing through the center of the hyperbola, which intersects the hyperbola at two points $\Omega$ and $\Omega^{\prime}$ provided that

$$
\lambda_{1} \lambda_{2} \kappa_{1} \kappa_{2}<0
$$

We shall see below that the sign of $(D)$ determines the existence or otherwise of the various categories of solitary waves.

(iii) The last curve $(P)=0$ is a set of two parallel lines if $\lambda_{1} \lambda_{2} \kappa_{1} \kappa_{2}<0$, or is empty if (19) is not satisfied. Moreover these two lines are tangent to $(H)$ precisely at $\Omega$ and $\Omega^{\prime}$. This curve locates the possible presence of a double solution for $k$ for a given real $c$, and hence locates the presence of those wavenumbers such that $c=c_{g}$.

The points $\Omega$ and $\Omega^{\prime}$ correspond to cases where 0 is a quadruple root of the dispersion equation, i.e. codimension 2 points for the reversible system. The general discussion of the unfolding may be found in [8], and this appears generically in the water-wave problem (see, for instance, section 3 of [10]).

A normal form analysis can now be carried out, based on the approach described, for instance, in [10]. Let us sum up the generic results:

1. Along $(H)=0$, in the half-plane where $\lambda_{1} \lambda_{2}(D)>0$, we denote by $L_{(H)}$ the above operator (13), which now has a double-zero eigenvalue, and two real eigenvalues (one $>0$ and one $<0$. Defining the eigenvector $\zeta_{0}$ and the generalized eigenvector $\zeta_{1}$ by

$$
\zeta_{0}=\left(\kappa_{1}, 0, c-\Delta_{1}, 0\right)^{t} \quad \text { and } \quad \zeta_{1}=\left(0, \kappa_{1}, 0, c-\Delta_{1}\right)^{t}
$$

the general theory (see, for instance, [10]) says that all "small" solutions are described in terms of a couple of real variables $(\alpha, \beta)$ by

$$
U=\alpha \zeta_{0}+\beta \zeta_{1}+\Psi\left(\alpha, \beta, c, \delta_{2}\right)
$$

meaning that they lie on a two-dimensional manifold (defined by $\Psi$ ) in the fourdimensional phase space. Moreover $(\alpha, \beta)$ satisfies the following two-dimensional system

$$
\begin{aligned}
& \frac{d \alpha}{d \xi}=\beta, \\
& \frac{d \beta}{d \xi}=\frac{(\widetilde{H})}{(D)} \alpha+a \alpha^{2}+f\left(\alpha, \beta, c, \delta_{2}\right)
\end{aligned}
$$


where $(\widetilde{H})=\frac{(D)}{2 \lambda_{1} \lambda_{2}}[(D)-\sqrt{(P)}] \sim(H), f=O(|\alpha|+\beta)^{3}, f$ is even in $\beta$, and the coefficient $a$ (which depends on $\left(c, \delta_{2}\right)$ ) may be computed explicitely. It can be proved that (provided that $a(0,0) \neq 0$ ) the above system possesses a solution homoclinic to 0 , for $(H)(D)>0$, which implies the existence of a pure solitary wave (homoclinic to 0 ) bifurcating on this side of $(H)=0$. For small amplitudes this solitary wave has the "sech" structure identified in Section 2 (see (5)).

2. Along $(H)=0$, and in the region $\lambda_{1} \lambda_{2}(D)<0$, the linear operator $L_{(H)}$ has a double-zero eigenvalue, and two imaginary eigenvalues $\pm i k_{0}$ where $\lambda_{1} \lambda_{2} k_{0}^{2}+(D)=0$. Let us define as above the eigenvector $\zeta_{0}$ and generalized eigenvector $\zeta_{1}$ belonging to the eigenvalue 0 , and now also the eigenvector $\zeta_{2}$ belonging to the eigenvalue $i k_{0}$. Then, the general theory (see, for instance, [10]) says that all small solutions are described by $(\alpha, \beta, \gamma)$ where, as above $\alpha, \beta$ are real variables, but $\gamma$ is a complex variable, and

$$
U=\alpha \zeta_{0}+\beta \zeta_{1}+\gamma \zeta_{2}+\bar{\gamma} \bar{\zeta}_{2}+\Psi\left(\alpha, \beta, \gamma, \bar{\gamma}, c, \delta_{2}\right)
$$

where $\Psi$ is a quadratic polynomial in $(\alpha, \beta, \gamma, \bar{\gamma})$, with coefficients smooth in $\left(c, \delta_{2}\right)$, and where

$$
\begin{aligned}
& \frac{d \alpha}{d \xi}=\beta, \\
& \frac{d \beta}{d \xi}=\frac{(\widetilde{H})}{(D)} \alpha+a \alpha^{2}+b|\gamma|^{2}+f\left(\alpha, \beta, \gamma, \bar{\gamma}, c, \delta_{2}\right) \\
& \frac{d \gamma}{d \xi}=i \gamma(k+d \alpha)+g\left(\alpha, \beta, \gamma, \bar{\gamma}, c, \delta_{2}\right)
\end{aligned}
$$

holds, with $a, b, d$ real and smooth in $\left(c, \delta_{2}\right)$ which can be computed easily, and $k=$ $k_{0}+O[(H)]$ is given by the dispersion relation, and

$$
(f, g)\left(\alpha,-\beta, \bar{\gamma}, \gamma, c, \delta_{2}\right)=(f,-\bar{g})\left(\alpha, \beta, \gamma, \bar{\gamma}, c, \delta_{2}\right), \quad|f|+|g|=O\left[(|\alpha|+|\beta|+|\gamma|)^{3}\right] .
$$

If we consider the above system with $f \equiv g \equiv 0$, we obtain in particular a oneparameter family of circles of homoclinic to periodic solutions, with $|\gamma|$ arbitrarily fixed between 0 and a quantity of order $O[(H)]$, and $\left.(\alpha, \beta) \rightarrow\left(\alpha_{\infty}(|\gamma|)\right), 0\right)$ as $\xi \rightarrow$ $\pm \infty$. The nonlinear coupling, due to $f$ and $g$ in the above system, between the oscillation produced by the eigenvalues $\pm i k$, and the slow exponential convergence to 0 of $(\alpha, \beta)$ given by $O\left(|(H)|^{1 / 2}\right)$, with $l$ a positive constant, leads to the impossibility of obtaining a pure homoclinic-to-zero orbit; that is there is no pure solitary wave in this case. Indeed, for the complete system, one can prove the existence of at least two homoclinic solutions to each of the periodic solutions whose principal part is given by

$$
\beta=0, \quad \frac{(\widetilde{H})}{(D)} \alpha+a \alpha^{2}+b|\gamma|^{2}=0, \quad|\gamma|=\text { constant }
$$


provided that $|\gamma|$ is not too small. A delicate mathematical analysis, made for instance in [4], proves that the minimal limiting amplitude $|\gamma|$ is exponentially small of order $O\left[\exp \left(-l|(H)|^{-1 / 2}\right)\right]$, with $l$ a positive constant, thus leading to the existence of bifurcating generalized solitary waves which tend to exponentially small oscillations at infinity.

3. Assume that $\lambda_{1} \lambda_{2} \kappa_{1} \kappa_{2}<0$. Along the line $(P)=0$, and in the region $\lambda_{1} \lambda_{2}(D)<0$, the linear operator $L_{(P)}$ has a pair of double eigenvalues $\pm i k_{1}$ such that $k_{1}^{2}=-(D) /\left(2 \lambda_{1} \lambda_{2}\right)$. This is the 1:1 resonance reversible Hopf bifurcation, which corresponds to the case $c=c_{g}$. Let us denote by $\zeta_{0}$ the eigenvector and $\zeta_{1}$ the generalized eigenvector such that $L_{(P)} \zeta_{0}=i k_{1} \zeta_{0}, L_{(P)} \zeta_{1}=i k_{1} \zeta_{1}+\zeta_{0}$, then the general theory (see for instance [10]) says that all small solutions are described by the complex variables $(\alpha, \beta)$ with

$$
U=\alpha \zeta_{0}+\beta \zeta_{1}+\bar{\alpha} \bar{\zeta}_{0}+\overline{\beta \zeta}_{1}+\Psi\left(\alpha, \beta, \bar{\alpha}, \bar{\beta}, c, \delta_{2}\right)
$$

where $\Psi$ is a cubic polynomial in $(\alpha, \beta, \bar{\alpha}, \bar{\beta})$ with coefficients smooth in $\left(c, \delta_{2}\right)$, and where

$$
\begin{array}{cc}
\frac{d \alpha}{d \xi}= & i k_{1} \alpha+\beta+i \alpha\left[\nu_{1}+p_{1}|\alpha|^{2}+\frac{i}{2} p_{2}(\alpha \bar{\beta}-\bar{\alpha} \beta)\right]+f, \\
\frac{d \beta}{d \xi}= & i k_{1} \beta+i \beta\left[\nu_{1}+p_{1}|\alpha|^{2}+\frac{i}{2} p_{2}(\alpha \bar{\beta}-\bar{\alpha} \beta)\right]+ \\
& +\alpha\left[\nu_{2}+q_{1}|\alpha|^{2}+\frac{i}{2} q_{2}(\alpha \bar{\beta}-\bar{\alpha} \beta)\right]+g, \\
\text { where } \quad(f, g)\left(\bar{\alpha},-\bar{\beta}, \alpha, \beta, c, \delta_{2}\right)=(-\bar{f}, \bar{g})\left(\alpha, \beta, \bar{\alpha}, \bar{\beta}, c, \delta_{2}\right), \\
|f|+|g|=O\left[(|\alpha|+|\beta|)^{4}\right], \quad \nu_{2} \sim\left[2 \lambda_{1} \lambda_{2}(D)\right]^{-1}(P), \quad \nu_{1} \sim\left(2 k_{1}\right)^{-1} \nu_{2} .
\end{array}
$$

Here $q_{1}<0$, at least near the codimension-two points $\Omega$ and $\Omega^{\prime}$ on the line $(P)=0$, thanks to the general result of [8]. We can see in the truncated system (without $f$ and $g)$ that the quantity $\frac{i}{2}(\alpha \bar{\beta}-\bar{\alpha} \beta)$ is a constant. A zero value of this first integral leads easily for $(P)<0$, to a "circle" of solutions homoclinic to zero, with damping oscillations at infinity. One can show (see [9]) that at least two homoclinic solutions persist for the full system (one with a maximum in the middle, the other with a trough). It results that on the side where $(P)<0$ (i.e. where the four eigenvalues of the linear operator $L(13)$ are complex), we have the bifurcation of two envelope solitary waves. Notice that if the sign of $q_{1}$ changes along $(P)=0$, one should obtain instead, in such a "defocusing" case, bifurcating "black" solitary waves on the side $(P)>0$.

Further, in this case $\mathbf{3}$, by contrast, near the lines $(P)=0$, on the side $\lambda_{1} \lambda_{2}(D)>0$, the usual conjecture is that, on the side $(P)<0$ we have the bifurcation diagram described by [3] (plethora of solitary waves). 
In figure $2 \mathrm{a}$ we show in the $\left(\delta_{2}, c\right)$ parameter plane the case $\kappa_{1} \kappa_{2}>0, \lambda_{1} \lambda_{2}>0$ (here $\lambda_{1}>0$ to fixing ideas). There are two little regions where the local analysis proves the existence of pure solitary waves (PSW), or generalized solitary waves (GSW), thus giving a rigorous understanding to figure 1a. We also plot the positions of the four eigenvalues $i k$, a cross means that the eigenvalue is double, while a dot means that it is a simple one. The case $\kappa_{1} \kappa_{2}<0, \lambda_{1} \lambda_{2}<0$ is analogous to this case, just rotated by $-\pi / 2$ in the parameter plane.

In figure $2 \mathrm{~b}$ we show the case $\kappa_{1} \kappa_{2}<0, \lambda_{1} \lambda_{2}>0$ (here again $\lambda_{1}>0$ to fixing ideas). In this figure we notice, in addition to the already mentioned bifurcating PSW and GSW, along half of the lines $(P)=0$, bifurcating envelope solitary waves (ESW) with oscillatory damping oscillations at infinity (with two types of symmetric waves). Next, near the two points $\Omega$ and $\Omega^{\prime}$ where 0 is a quadruple eigenvalue, we have on the sides between the two lines $(P)=0$, a plethora of solitary waves of the types described in [3], in a region connecting the region ESW with the half lines $(P)=0$ where there are two double real eigenvalues.

In figure $2 \mathrm{c}$ we show the case $\kappa_{1} \kappa_{2}>0, \lambda_{1} \lambda_{2}<0$, with $\lambda_{1}>0$ and $\lambda_{1}+\lambda_{2}>0$ for fixing ideas. Since $\lambda_{1} \lambda_{2} \kappa_{1} \kappa_{2}<0$ this figure is analogous to figure 2b. The connection with figure $1 \mathrm{~b}, \mathrm{c}$ is as follows. If we make $\delta_{1}=0$, and consider $\delta_{2}>0$ fixed large enough, we give a rigorous understanding of figure $1 \mathrm{~b}$, while figure $1 \mathrm{c}$ would correspond to a situation analogous to that shown here when considering a cut by $\delta_{2}<0$ fixed small enough. Indeed, in the case of figure $1 \mathrm{c}$ we have $\kappa_{1} \kappa_{2}>0, \lambda_{1} \lambda_{2}<0$, but $\lambda_{1}+\lambda_{2}<0$ which changes the sides where $(D)>0$ or $<0$, hence permuting the GSW and PSW regions.

\section{References}

[1] M.J. Ablowitz and H.Segur. Solitons and the inverse scattering transform. SIAM studies in Applied Mathematics, SIAM, Philadelphia (1981), 425pp.

[2] T.R. Akylas. Envelope solitary waves with stationary crests. Phys. Fluids (1993), 789-791.

[3] A.R. Champneys and J.F. Toland. Bifurcation of a plethora of large amplitude homoclinic orbits for autonomous Hamiltonian systems. Nonlinearity 6 (1993), 665-721.

[4] E.Lombardi. Oscillatory integrals and phenomena beyond all algebraic orders, with applications to homoclinic orbits in reversible systems. Lecture Notes in Maths, 1741 (2000), Springer. 
[5] R. Grimshaw, B. Malomed and E. Benilov. Solitary waves with damped oscillatory tails: an analysis of the fifth-order Korteweg de-Vries equation. Physica D, 77 (1994), 473-485.

[6] R. Grimshaw and N. Joshi. Weakly non-local solitary waves in a singularly perturbed Korteweg-de Vries equation. SIAM J. Appl. Math., 55 (1995), 124-135.

[7] G. Iooss and K. Kirchgassner. Bifurcation d'ondes solitaires en présence d'une faible tension superficielle. C. R. Acad. Sci. paris 311 (1990), 265-268.

[8] G. Iooss. A codimension 2 bifurcation for reversible vector fields. Fields Institute Com., 4 (1995), 201-217.

[9] G.Iooss, M.C.Pérouème. Perturbed homoclinic solutions in reversible 1:1 resonance vector fields. J. Differential Equations, 102 (1993), 62-88.

[10] F. Dias and G.Iooss. Water-waves as a spatial dynamical system. Handbook for Mathematical Fluid Dynamics. eds. S.Friedlander, D.Serre, Elsevier 62pp (to appear).

[11] R. Grimshaw. Models for long-wave instability due to a resonance between two waves. Trends in Applications of Mathematics to Mechanics", ed. G. Iooss, O. Gues and A. Nouri, Monographs and Surveys in Pure and Applied Mathematics, 106, Chapman \&f Hall/CRC (2000), 183-192.

[12] R. Grimshaw. Internal solitary waves. Environmental Stratified Flows, ed. R. Grimshaw, Kluwer, Massachusetts, Chapter 1, 1-30 (to appear).

[13] R. Grimshaw and P. Cook. Solitary waves with oscillatory tails. Proceedings of Second International Conference on Hydrodynamics, Hong Kong, 1996, "Hydrodynamics: Theory and Applications", Vol. 1, ed. A.T. Chwang, J.H.W. Lee and D.Y.C. Leung, A.A. Balkema, Rotterdam (1996), 327-336. 

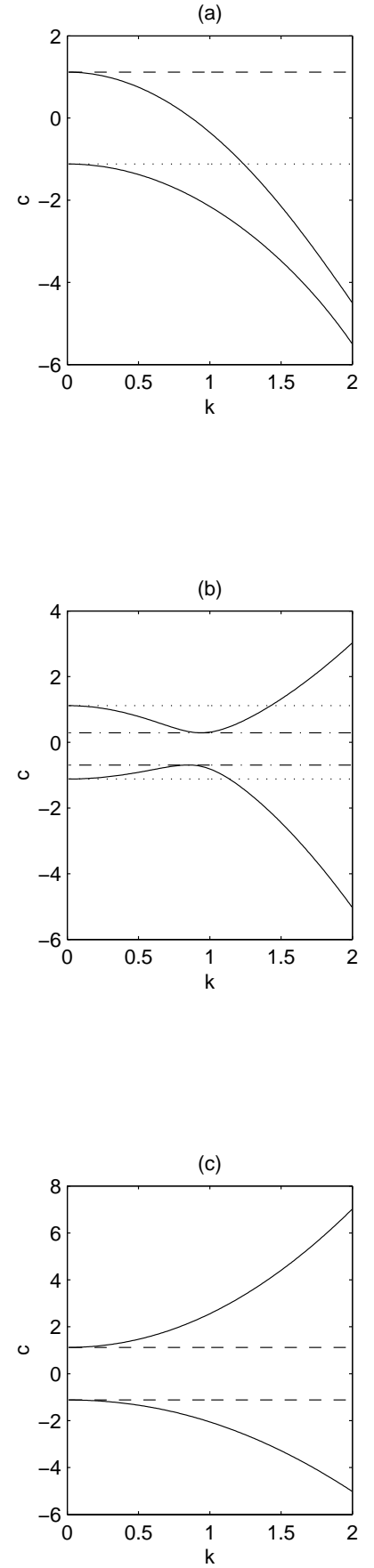

Figure 1: The dispersion relation (4) for the parameter setting $\Delta_{1}=-\Delta_{2}=1.0, \kappa_{1,2}=0.5$ and $\lambda_{1,2}$ given by (a) $1.5,1.0$, (b) $1.5,-1.0$ and (c) $-1.5,1.0$. In each case the dashed, dotted or dash-dot lines indicate a bifurcation to a pure solitary wave, a generalized solitary wave or to an envelope solitary wave respectively. 
(a)

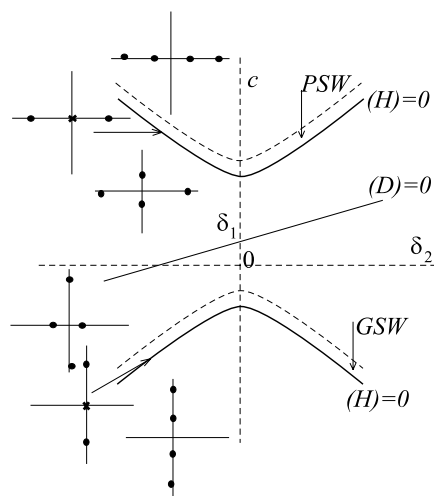

(b)

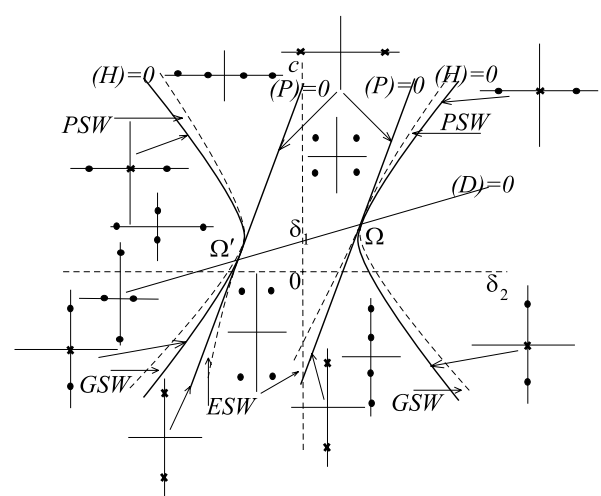

(c)

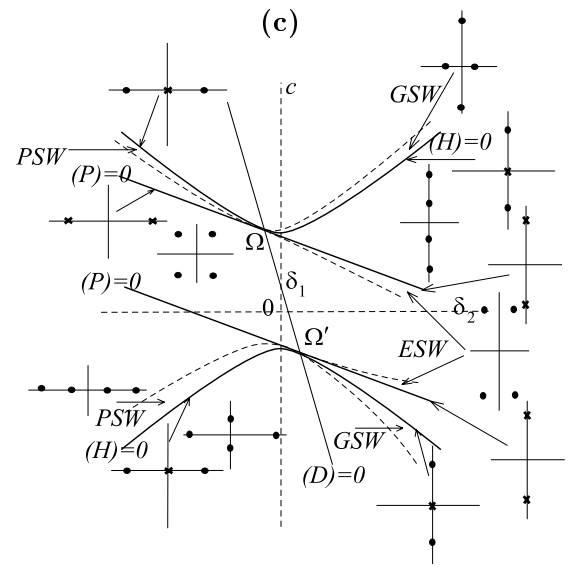

Figure 2: Location of bifurcations in the $\left(\delta_{2}, c\right)$-parameter plane, and the positions of the eigenvalues $\sigma=i k$ : (a) the case $\kappa_{1} \kappa_{2}>0$ and $\lambda_{1,2}>0$; (b) the case $\kappa_{1} \kappa_{2}<0$ and $\lambda_{1,2}>0$; (c) the case $\kappa_{1} \kappa_{2}>0, \lambda_{1}>0>\lambda_{2}$ with $\lambda_{1}+\lambda_{2}>0$. 